\title{
ESTUDO COMPARATIVO DOS HIDROCARBONETOS EMITIDOS PELO GÁS DE ESCAPAMENTO DE UM VEÍCULO FLEX QUANDO ABASTECIDO COM E22 E COM E100.
}

\author{
Laerte Graner; Irineu Secolo Garcia; Henry Joseph Jr.
}

Volkswagen do Brasil

\section{RESUMO:}

O presente trabalho tem como objetivo qualificar, quantificar e comparar os hidrocarbonetos emitidos pelo gás de escapamento de um veículo flex fuel, quando abastecido com $100 \%$ de gasolina tipo C (E22) e com 100\% de etanol hidratado combustível (E100), visando comparar a reatividade específica do potencial de formação de ozônio atmosférico, dos gases provenientes destes dois combustíveis.

A análise das emissões dos hidrocarbonetos foi feita por cromatografia gasosa, em analogia ao procedimento "California NON-Methane Organic Gas Test procedures - March 22, 2012" do CARB (California Air Resources Board).

As medições dos gases de escapamento foram realizadas durante ensaios dinamométricos de chassis conforme norma NBR 6601.

\section{INTRODUÇÃO:}

Em função da inexistência no Brasil, de método, de equipamento de análise e de conhecimento para qualificar, quantificar e comparar os gases emitidos pelo gás de escapamento de um veículo flex fuel, quando abastecido com $100 \% \mathrm{com}$ etanol hidratado (E100) e com 100\% de gasolinaC22, a Volkswagen do Brasil realizou um estudo de detalhamento das emissões de NMHC emitidas pelo gás de escapamento, utilizando a técnica analítica por cromatografia gasosa por dessorção térmica e objetivando determinar a reatividade específica do potencial de formação de ozônio atmosférico dos gases provenientes destes dois combustíveis. O referido detalhamento foi realizado no Laboratório de Emissões da Volkswagen na Alemanha em Wolfsburg.

Para o cálculo da reatividade dos NMHC foi empregado parte do conceito do California NONMethane Organic Gas Test procedures - March 22, 2012) do CARB (California Air Resources Board).

\section{METODOLOGIAS UTILIZADAS:}

\subsection{PROCEDIMENTOS DE AMOSTRAGEM}

A amostragem dos gases para análise, procedentes dos gás de escapamento, foi feita através de um dispositivo que transfere parte dos gases contidos nos bags do CVS para pequenos bags de $25 \mathrm{~L}$. Esta transferência ocorreu imediatamente após o término de um ciclo completo de emissões conforme NBR 6601.

\subsection{PROCEDIMENTO DE ANÁLISE}


Método de análise: a análise dos pequenos bags contendo as emissões hidrocarbonetos foi feita por cromatografia gasosa com dessorção térmica, com detetor FID, em analogia ao procedimento "California NON-Methane Organic Gas Test procedures - March 22, 2012" do CARB (California Air Resources Board).

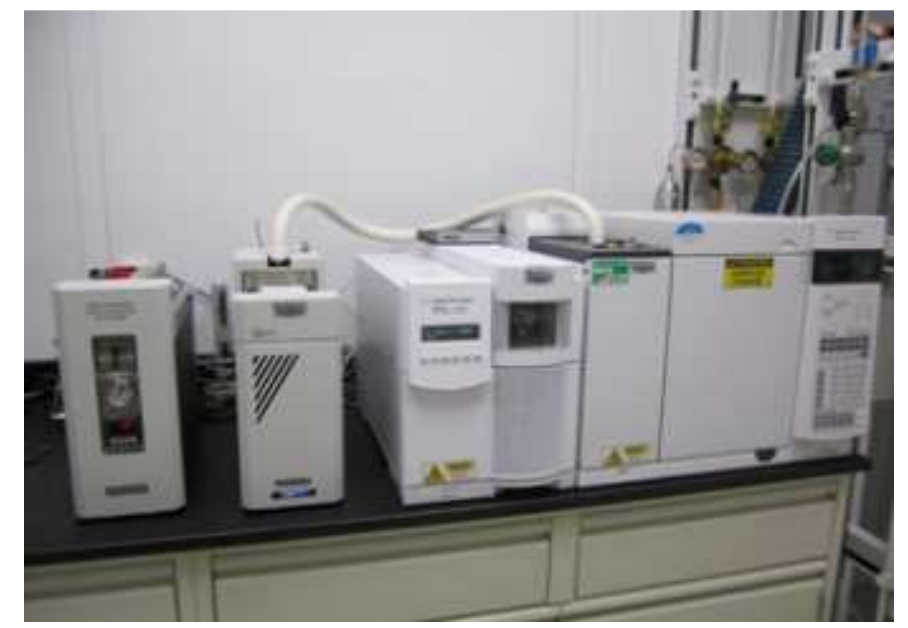

Figura nำ 1 - Equipamento de análise por cromatografia gasosa por dessorção térmica

\section{CARACTERÍSTICAS DO VEÍCULO UTILIZADO E DO ENSAIO:}

Para estas avalições foi utilizado o veículo Polo BlueMotion 1.6L EFlex de fabricação brasileira modelo ano 2012/2013 com aproximadamente $5650 \mathrm{~km}$ de rodagem. O veículo foi testado em dinamômetro de chassis conforme o ciclo de ensaio da NBR 6601. Para a aprendizagem do veículo em relação aos combustíveis, foi feito um ensaio de condicionamento antes de cada ensaio conforme NBR 6601.

\section{COMBUSTÍVEIS UTILIZADOS NOS ENSAIOS:}

Os combustíveis foram adquiridos no Brasil e exportadas para a Volkswagen em Wolfsburg na Alemanha.

\subsection{CARACTERÍSTICAS FÍSICO QUÍMICAS DA GASOLINA C22 (E22):}

Ver tabela no 1 anexa

\subsection{CARACTERÍSTICAS FÍSICO QUÍMICAS DO ETANOL HIDRATADO (E100):}

Ver tabela nำ 2 anexa.

\section{ENSAIOS E RESULTADOS}

\subsection{CROMATOGRAMAS}


CROMATOGRAMAS DA FASE 1

E22

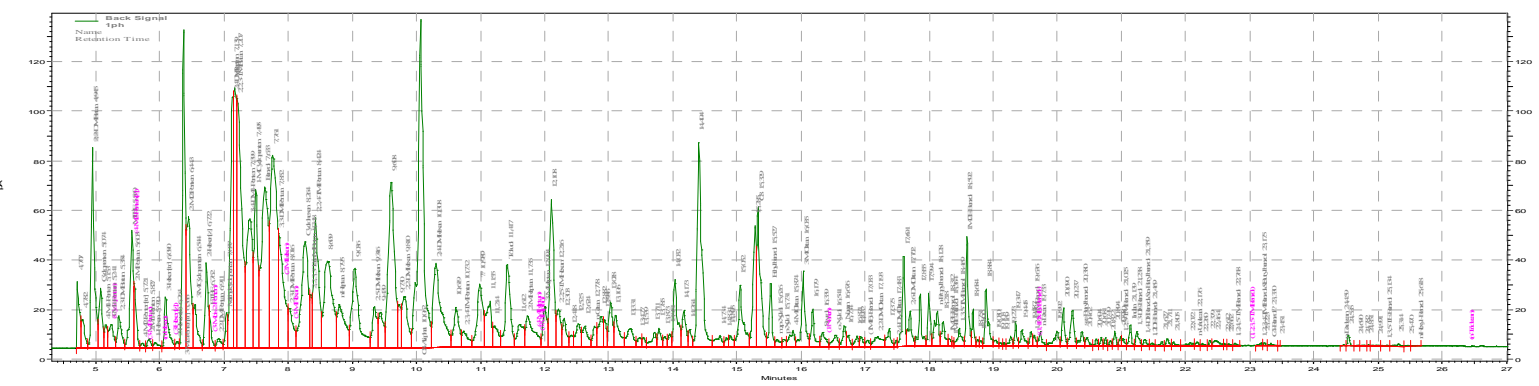

E100

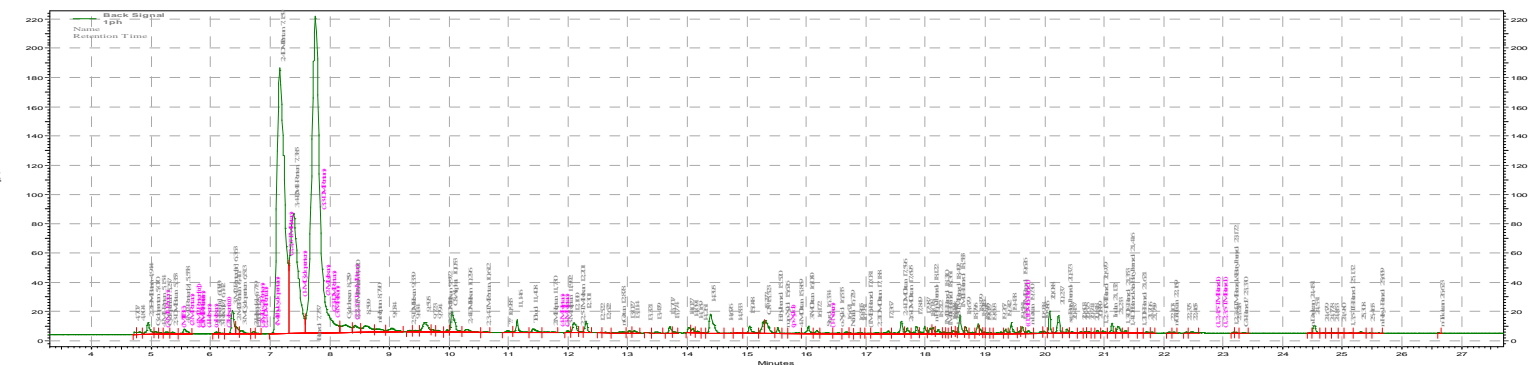

CROMATOGRAMAS DA FASE 2

E22

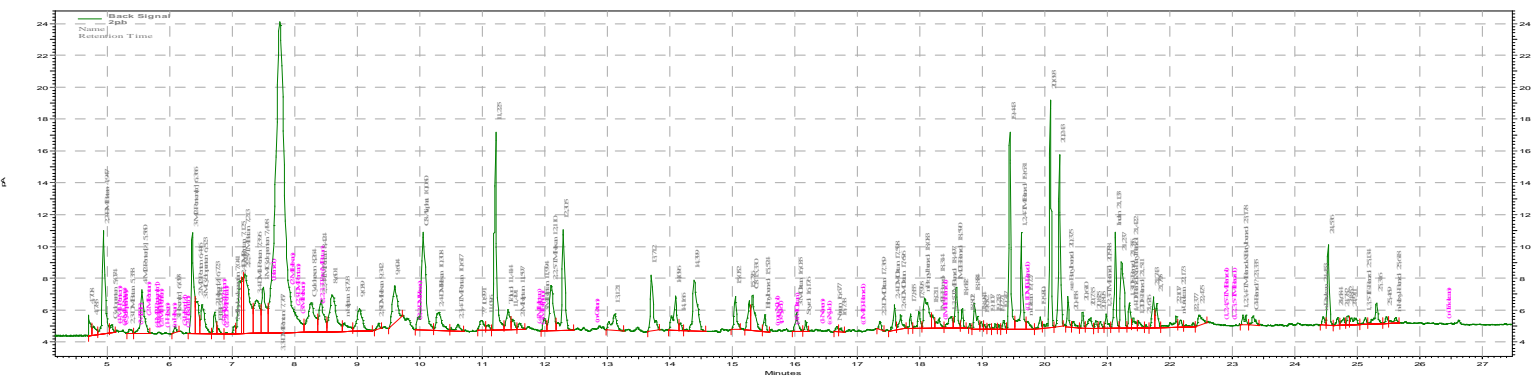

E100

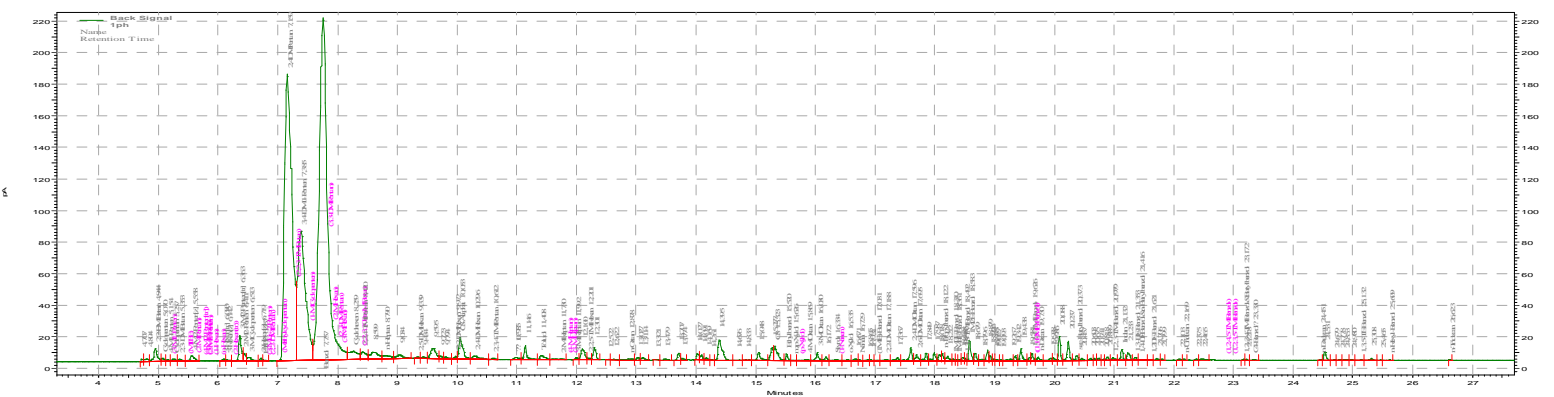


CROMATOGRAMA DA FASE 3

E22

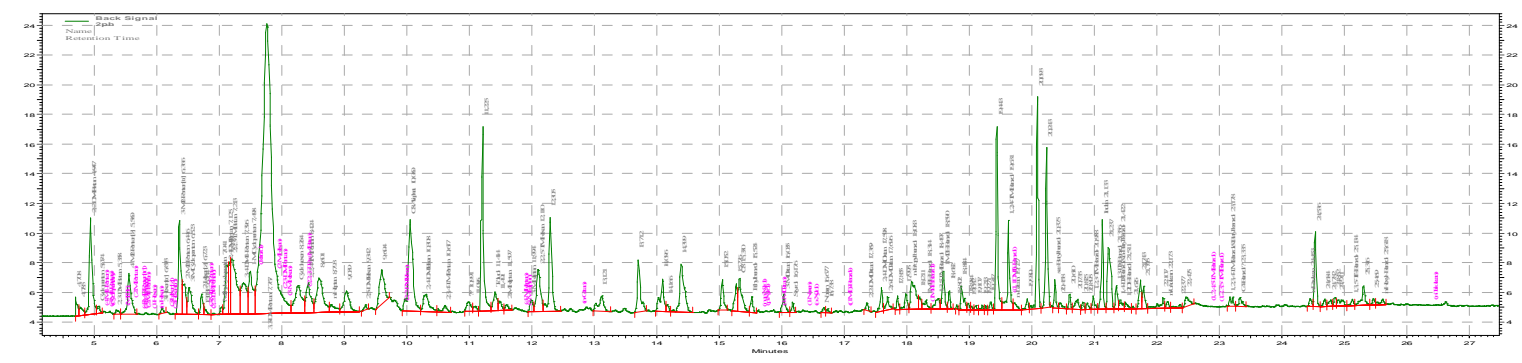

E100

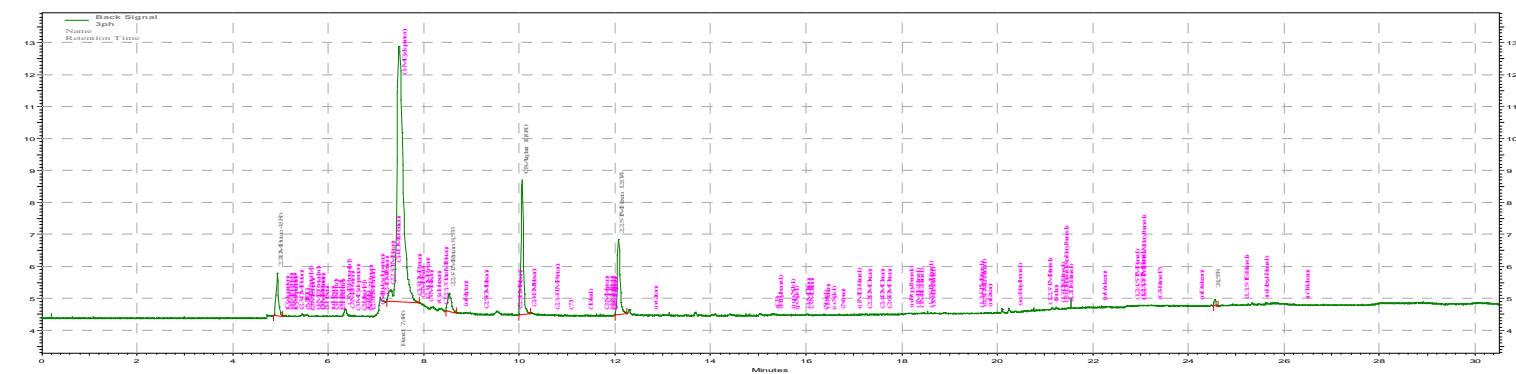

CROMATOGRAMAS AR DILUIÇÃO

E22

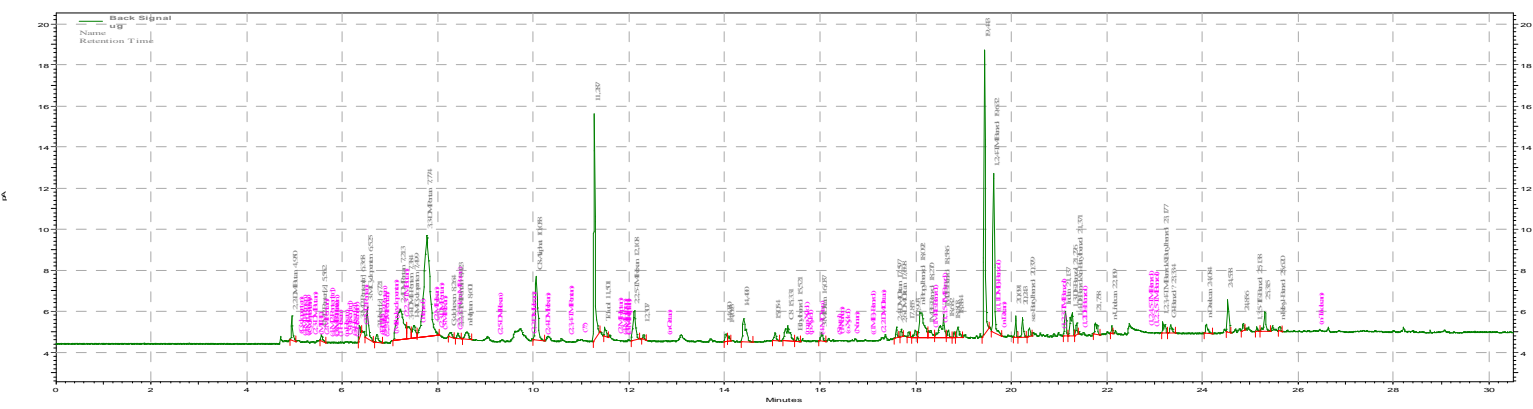

E100

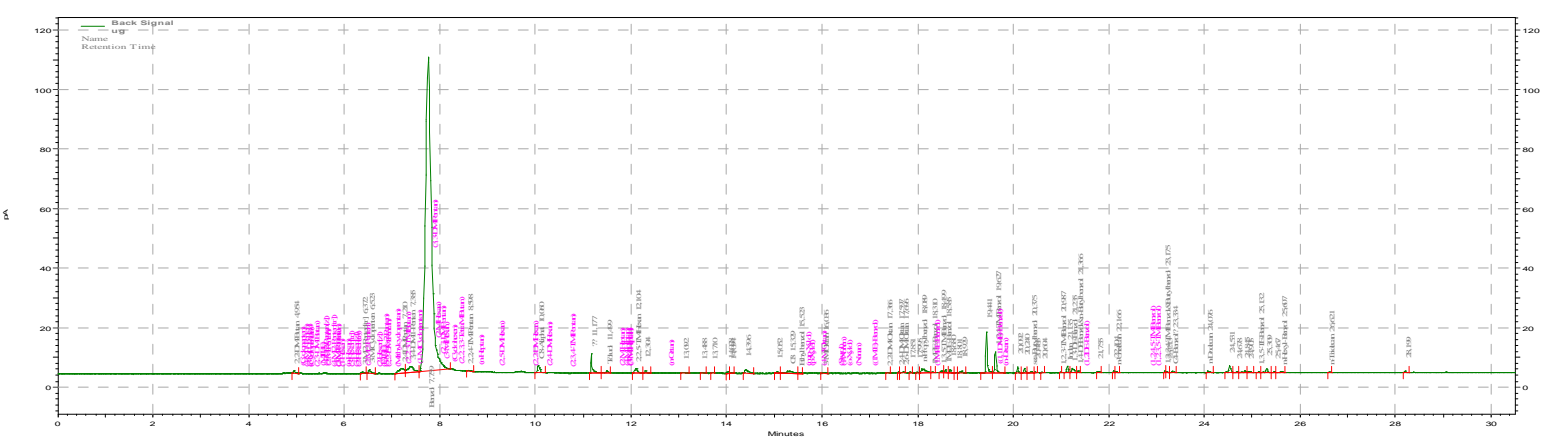




\subsection{RESULTADOS DAS MEDIÇÕES DE NMHC E DO OFP:}

\section{PARTE 1}

\begin{tabular}{|c|c|c|c|c|c|c|c|c|c|c|}
\hline \multirow[b]{2}{*}{$\begin{array}{l}\text { Hidrocarbonetos } \\
\text { (C2-C12) }\end{array}$} & \multirow[b]{2}{*}{$\begin{array}{c}\text { Mess- } \\
\text { verfahren }\end{array}$} & \multirow[b]{2}{*}{ MIR } & \multicolumn{2}{|c|}{ 0404-E22 } & \multicolumn{2}{|c|}{ 0405-E22 } & \multicolumn{2}{|c|}{ 0410-E100 } & \multicolumn{2}{|c|}{ 0411-E100 } \\
\hline & & & $\begin{array}{l}\mathrm{NMHC} \\
\mathrm{mg} / \mathrm{km}\end{array}$ & \begin{tabular}{|c|} 
OFP \\
$\mathrm{mg} / \mathrm{km}$
\end{tabular} & \begin{tabular}{c|}
$\mathrm{NMHC}$ \\
$\mathrm{mg} / \mathrm{km}$
\end{tabular} & \begin{tabular}{|c|} 
OFP \\
$\mathrm{mg} / \mathrm{km}$
\end{tabular} & \begin{tabular}{l|}
$\mathrm{NMHC}$ \\
$\mathrm{mg} / \mathbf{k m}$
\end{tabular} & \begin{tabular}{|c|} 
OFP \\
$\mathrm{mg} / \mathrm{km}$
\end{tabular} & $\begin{array}{l}\mathrm{NMHC} \\
\mathrm{mg} / \mathrm{km}\end{array}$ & $\begin{array}{l}\text { OFP } \\
\mathrm{mg} / \mathrm{km}\end{array}$ \\
\hline Ethan & VocAir & 0.28 & 1.319 & 0.369 & 1.129 & 0.316 & 0.986 & 0.276 & 0.854 & 0.239 \\
\hline Ethen & VocAir & 9.00 & 2.354 & 21.187 & 2.386 & 21.477 & 5.140 & 46.262 & 3.589 & 32.300 \\
\hline Propan & VocAir & 0.49 & 0.050 & 0.024 & 0.066 & 0.032 & 0.027 & 0.013 & 0.035 & 0.017 \\
\hline Propen & VocAir & 11.66 & 0.940 & 10.964 & 1.015 & 11.837 & 0.131 & 1.522 & 0.084 & 0.976 \\
\hline Acetylen & VocAir & 0.95 & 0.097 & 0.092 & 0.169 & 0.160 & 0.262 & 0.249 & 0.188 & 0.179 \\
\hline 2-Methyl-Propan & VocAir & 1.23 & 0.255 & 0.313 & 0.358 & 0.440 & 0.904 & 1.112 & 0.636 & 0.783 \\
\hline n-Butan & VocAir & 1.15 & 0.434 & 0.499 & 0.629 & 0.723 & 0.296 & 0.341 & 0.113 & 0.130 \\
\hline trans-2-Buten & VocAir & 15.16 & 0.121 & 1.836 & 0.132 & 2.005 & 0.046 & 0.700 & 0.027 & 0.413 \\
\hline 1-Buten & VocAir & 9.73 & 0.283 & 2.756 & 0.508 & 4.944 & 0.133 & 1.296 & 0.153 & 1.492 \\
\hline 2-Methyl-Propen & VocAir & 6.29 & 0.271 & 1.703 & 0.431 & 2.714 & 0.030 & 0.188 & 0.025 & 0.160 \\
\hline cis-2-Buten & VocAir & 14.24 & 0.092 & 1.313 & 0.103 & 1.460 & 0.071 & 1.012 & 0.046 & 0.651 \\
\hline 1,2-Propadien & VocAir & 8.45 & 0.000 & 0.000 & 0.000 & 0.000 & 0.000 & 0.000 & 0.000 & 0.000 \\
\hline Cyclo-Pentan & VocAir & 2.39 & 0.000 & 0.000 & 0.000 & 0.000 & 0.000 & 0.000 & 0.000 & 0.000 \\
\hline 2-Methyl-Butan & VocAir & 1.45 & 1.370 & 1.986 & 1.802 & 2.612 & 0.447 & 0.648 & 0.034 & 0.050 \\
\hline n-Pentan & VocAir & 1.31 & 0.784 & 1.027 & 1.114 & 1.459 & 0.175 & 0.229 & 0.074 & 0.098 \\
\hline Propin & VocAir & 6.72 & 0.058 & 0.388 & 0.088 & 0.595 & 0.010 & 0.070 & 0.006 & 0.038 \\
\hline 2,2-Dimethyl-Propan & VocAir & 0.67 & 0.000 & 0.000 & 0.000 & 0.000 & 0.000 & 0.000 & 0.000 & 0.000 \\
\hline 1,3-Butadien & VocAir & 12.61 & 0.317 & 3.995 & 0.266 & 3.358 & 0.022 & 0.277 & 0.016 & 0.200 \\
\hline 3-Methyl-1-Buten & VocAir & 6.99 & 0.000 & 0.000 & 0.000 & 0.000 & 0.000 & 0.000 & 0.000 & 0.000 \\
\hline Cyclo-Penten & VocAir & 6.77 & 0.000 & 0.000 & 0.000 & 0.000 & 0.000 & 0.000 & 0.000 & 0.000 \\
\hline trans-2-Penten & VocAir & 10.56 & 0.261 & 2.754 & 0.307 & 3.247 & 0.048 & 0.507 & 0.014 & 0.151 \\
\hline 2-Methyl-2-Buten & VocAir & 14.08 & 0.370 & 5.214 & 0.397 & 5.583 & 0.054 & 0.754 & 0.019 & 0.273 \\
\hline 1-Penten & VocAir & 7.21 & 0.000 & 0.000 & 0.000 & 0.000 & 0.000 & 0.000 & 0.000 & 0.000 \\
\hline 2-Methyl-1-Buten & VocAir & 6.40 & 0.000 & 0.000 & 0.000 & 0.000 & 0.000 & 0.000 & 0.000 & 0.000 \\
\hline cis-2-Penten & VocAir & 10.38 & 0.088 & 0.912 & 0.103 & 1.069 & 0.015 & 0.160 & 0.004 & 0.045 \\
\hline 2-Methyl-1,3-Butadien & VocAir & 10.61 & 0.235 & 2.496 & 0.187 & 1.986 & 0.015 & 0.158 & 0.018 & 0.187 \\
\hline 1-Butin & VocAir & 6.11 & 0.171 & 1.045 & 0.197 & 1.201 & 0.022 & 0.136 & 0.008 & 0.051 \\
\hline 2-Butin & VocAir & 16.32 & 0.114 & 1.869 & 0.135 & 2.201 & 0.020 & 0.324 & 0.006 & 0.100 \\
\hline 1-Buten-3-in & VocAir & 10.48 & 0.000 & 0.000 & 0.000 & 0.000 & 0.000 & 0.000 & 0.000 & 0.000 \\
\hline 1,3-Cyclo-Pentadien & VocAir & 6.98 & 0.000 & 0.000 & 0.000 & 0.000 & 0.000 & 0.000 & 0.000 & 0.000 \\
\hline 3,3-Dimethyl-1-Buten & VocAir & 5.82 & 0.000 & 0.000 & 0.000 & 0.000 & 0.000 & 0.000 & 0.000 & 0.000 \\
\hline 2,2-Dimethyl-Butan & VocAir & 1.17 & 0.000 & 0.000 & 0.000 & 0.000 & 0.000 & 0.000 & 0.000 & 0.000 \\
\hline 4-Methyl-1-Penten & VocAir & 5.68 & 0.000 & 0.000 & 0.000 & 0.000 & 0.000 & 0.000 & 0.000 & 0.000 \\
\hline 3-Methyl-1-Penten & VocAir & 6.14 & 0.000 & 0.000 & 0.000 & 0.000 & 0.000 & 0.000 & 0.000 & 0.000 \\
\hline 2,3-Dimethyl-Butan & VocAir & 0.97 & 0.000 & 0.000 & 0.000 & 0.000 & 0.000 & 0.000 & 0.000 & 0.000 \\
\hline 4-Methyl-cis-2-Penten & VocAir & 8.12 & 0.000 & 0.000 & 0.000 & 0.000 & 0.000 & 0.000 & 0.000 & 0.000 \\
\hline 2-Methyl-Pentan & VocAir & 1.50 & 0.135 & 0.203 & 0.196 & 0.295 & 0.000 & 0.000 & 0.000 & 0.000 \\
\hline
\end{tabular}

OFP = Ozone Forming Potencial $(\mathrm{mg} / \mathrm{km})$ 


\section{PARTE 2}

\begin{tabular}{|c|c|c|c|c|c|c|c|c|c|c|}
\hline \multirow[b]{2}{*}{$\begin{array}{l}\text { Hidrocarbonetos } \\
\text { (C2-C12) }\end{array}$} & \multirow[b]{2}{*}{$\begin{array}{c}\text { Mess- } \\
\text { verfahren }\end{array}$} & \multirow[b]{2}{*}{ MIR } & \multicolumn{2}{|c|}{ 0404-E22 } & \multicolumn{2}{|c|}{ 0405-E22 } & \multicolumn{2}{|c|}{ 0410-E100 } & \multicolumn{2}{|c|}{ 0411-E100 } \\
\hline & & & $\begin{array}{l}\mathrm{NMHC} \\
\mathrm{mg} / \mathrm{km}\end{array}$ & $\begin{array}{c}\text { OFP } \\
\mathrm{mg} / \mathrm{km}\end{array}$ & $\begin{array}{l}\mathrm{NMHC} \\
\mathrm{mg} / \mathrm{km}\end{array}$ & \begin{tabular}{|c|} 
OFP \\
$\mathrm{mg} / \mathrm{km}$
\end{tabular} & $\begin{array}{l}\mathrm{NMHC} \\
\mathrm{mg} / \mathrm{km}\end{array}$ & $\begin{array}{l}\text { OFP } \\
\mathrm{mg} / \mathrm{km}\end{array}$ & \begin{tabular}{l|}
$\mathrm{NMHC}$ \\
$\mathrm{mg} / \mathrm{km}$
\end{tabular} & $\begin{array}{l}\text { OFP } \\
\mathrm{mg} / \mathrm{km}\end{array}$ \\
\hline 4-Methyl-trans-2-Penten & VocAir & 8.12 & 0.000 & 0.000 & 0.000 & 0.000 & 0.000 & 0.000 & 0.000 & 0.000 \\
\hline 3-Methyl-Pentan & VocAir & 1.80 & 0.000 & 0.000 & 0.000 & 0.000 & 0.000 & 0.000 & 0.000 & 0.000 \\
\hline 2-Methyl-1-Penten & VocAir & 5.26 & 0.000 & 0.000 & 0.000 & 0.000 & 0.000 & 0.000 & 0.000 & 0.000 \\
\hline 1-Hexen & VocAir & 5.49 & 0.014 & 0.076 & 0.017 & 0.092 & 0.000 & 0.000 & 0.000 & 0.000 \\
\hline n-Hexan & VocAir & 1.24 & 0.008 & 0.010 & 0.000 & 0.000 & 0.000 & 0.000 & 0.000 & 0.000 \\
\hline trans-3-Hexen & VocAir & 7.57 & 0.000 & 0.000 & 0.000 & 0.000 & 0.000 & 0.000 & 0.000 & 0.000 \\
\hline cis-3-Hexen & VocAir & 7.61 & 0.000 & 0.000 & 0.000 & 0.000 & 0.000 & 0.000 & 0.000 & 0.000 \\
\hline trans-2-Hexen & VocAir & 8.62 & 0.018 & 0.159 & 0.025 & 0.216 & 0.000 & 0.000 & 0.000 & 0.000 \\
\hline 3-Methyl-trans-2-Penten & VocAir & 13.17 & 0.000 & 0.000 & 0.000 & 0.000 & 0.000 & 0.000 & 0.000 & 0.000 \\
\hline 2-Methyl-2-Penten & VocAir & 11.00 & 0.000 & 0.000 & 0.000 & 0.000 & 0.000 & 0.000 & 0.000 & 0.000 \\
\hline 3-Methyl-Cyclo-Penten & VocAir & 5.10 & 0.000 & 0.000 & 0.000 & 0.000 & 0.000 & 0.000 & 0.000 & 0.000 \\
\hline cis-2-Hexen & VocAir & 8.31 & 0.326 & 2.710 & 0.472 & 3.919 & 0.021 & 0.174 & 0.008 & 0.066 \\
\hline 3-Methyl-cis-2-Penten & VocAir & 12.49 & 0.000 & 0.000 & 0.000 & 0.000 & 0.000 & 0.000 & 0.000 & 0.000 \\
\hline 2,2-Dimethyl-Pentan & VocAir & 1.12 & 0.000 & 0.000 & 0.000 & 0.000 & 0.000 & 0.000 & 0.000 & 0.000 \\
\hline Methyl-Cyclo-Pentan & VocAir & 2.19 & 0.000 & 0.000 & 0.000 & 0.000 & 0.000 & 0.000 & 0.000 & 0.000 \\
\hline 2,4-Dimethyl-Pentan & VocAir & 1.55 & 0.000 & 0.000 & 0.000 & 0.000 & 0.000 & 0.000 & 0.000 & 0.000 \\
\hline 2,2,3-Trimethyl-Butan & VocAir & 1.11 & 0.000 & 0.000 & 0.000 & 0.000 & 0.000 & 0.000 & 0.000 & 0.000 \\
\hline 3,4-Dimethyl-Penten & VocAir & 4.84 & 0.000 & 0.000 & 0.000 & 0.000 & 0.000 & 0.000 & 0.000 & 0.000 \\
\hline 1-Methyl-Cyclo-Penten & VocAir & 12.49 & 0.000 & 0.000 & 0.000 & 0.000 & 0.000 & 0.000 & 0.000 & 0.000 \\
\hline Benzol & VocAir & 0.72 & 1.189 & 0.856 & 1.620 & 1.167 & 8.770 & 6.314 & 10.027 & 7.219 \\
\hline 3-Methyl-1-Hexen & VocAir & 4.56 & 0.000 & 0.000 & 0.000 & 0.000 & 0.000 & 0.000 & 0.000 & 0.000 \\
\hline 3,3-Dimethyl-Pentan & VocAir & 1.20 & 0.000 & 0.000 & 0.000 & 0.000 & 0.000 & 0.000 & 0.000 & 0.000 \\
\hline Cyclo-Hexan & VocAir & 1.25 & 0.000 & 0.000 & 0.000 & 0.000 & 0.000 & 0.000 & 0.000 & 0.000 \\
\hline 2-Methyl-Hexan & VocAir & 1.19 & 0.000 & 0.000 & 0.000 & 0.000 & 0.000 & 0.000 & 0.000 & 0.000 \\
\hline 2,3-Dimethyl-Pentan & VocAir & 1.34 & 0.000 & 0.000 & 0.000 & 0.000 & 0.000 & 0.000 & 0.000 & 0.000 \\
\hline 3-Methyl-Hexan + Cyclo-Hexen & VocAir & 1.61 & 0.832 & 1.339 & 1.069 & 1.721 & 0.400 & 0.644 & 0.103 & 0.165 \\
\hline cis-1,3-Dimethyl-Cyclo-Pentan & VocAir & 1.94 & 0.000 & 0.000 & 0.000 & 0.000 & 0.000 & 0.000 & 0.000 & 0.000 \\
\hline 3-Ethyl-Pentan & VocAir & 1.90 & 0.000 & 0.000 & 0.000 & 0.000 & 0.000 & 0.000 & 0.000 & 0.000 \\
\hline trans-1,3-Dimethyl-Cyclo-Pentan & VocAir & 1.94 & 0.000 & 0.000 & 0.000 & 0.000 & 0.000 & 0.000 & 0.000 & 0.000 \\
\hline 1-Hepten & VocAir & 4.43 & 0.000 & 0.000 & 0.000 & 0.000 & 0.000 & 0.000 & 0.000 & 0.000 \\
\hline 2,2,4-Trimethyl-Pentan & VocAir & 1.26 & 0.871 & 1.097 & 0.789 & 0.995 & 0.202 & 0.254 & 0.350 & 0.441 \\
\hline trans-3-Hepten & VocAir & 6.32 & 0.000 & 0.000 & 0.000 & 0.000 & 0.000 & 0.000 & 0.000 & 0.000 \\
\hline n-Heptan & VocAir & 1.07 & 0.318 & 0.340 & 0.419 & 0.449 & 0.192 & 0.205 & 0.089 & 0.095 \\
\hline 2-Methyl-2-Hexen & VocAir & 9.47 & 0.000 & 0.000 & 0.000 & 0.000 & 0.000 & 0.000 & 0.000 & 0.000 \\
\hline 3-Methyl-trans-3-Hexen & VocAir & 9.72 & 0.000 & 0.000 & 0.000 & 0.000 & 0.000 & 0.000 & 0.000 & 0.000 \\
\hline trans-2-Hepten & VocAir & 7.14 & 0.000 & 0.000 & 0.000 & 0.000 & 0.000 & 0.000 & 0.000 & 0.000 \\
\hline 3-Ethyl-2-Penten & VocAir & 9.75 & 0.000 & 0.000 & 0.000 & 0.000 & 0.000 & 0.000 & 0.000 & 0.000 \\
\hline 2,4,4-Trimethyl-1-Penten & VocAir & 3.34 & 0.000 & 0.000 & 0.000 & 0.000 & 0.000 & 0.000 & 0.000 & 0.000 \\
\hline 2,3-Dimethyl-2-Penten & VocAir & 9.74 & 0.000 & 0.000 & 0.000 & 0.000 & 0.000 & 0.000 & 0.000 & 0.000 \\
\hline cis-2-Hep & VocAir & 7.16 & 0.000 & 0.000 & 0.000 & 0.000 & 0.000 & 0.000 & 0.000 & 0.000 \\
\hline Methyl-Cyclo-Hexan & VocAir & 1.70 & 0.000 & 0.000 & 0.000 & 0.000 & 0.000 & 0.000 & 0.000 & 0.000 \\
\hline
\end{tabular}




\section{PARTE 3}

\begin{tabular}{|c|c|c|c|c|c|c|c|c|c|c|}
\hline \multirow[b]{2}{*}{$\begin{array}{l}\text { Hidrocarbonetos } \\
\text { (C2-C12) }\end{array}$} & \multirow[b]{2}{*}{$\begin{array}{c}\text { Mess- } \\
\text { verfahren }\end{array}$} & \multirow[b]{2}{*}{ MIR } & \multicolumn{2}{|c|}{ 0404-E22 } & \multicolumn{2}{|c|}{ 0405-E22 } & \multicolumn{2}{|c|}{ 0410-E100 } & \multicolumn{2}{|c|}{ 0411-E100 } \\
\hline & & & $\begin{array}{l}\mathrm{NMHC} \\
\mathrm{mg} / \mathrm{km}\end{array}$ & \begin{tabular}{|c|} 
OFP \\
$\mathrm{mg} / \mathrm{km}$
\end{tabular} & $\begin{array}{l}\mathrm{NMHC} \\
\mathrm{mg} / \mathrm{km}\end{array}$ & \begin{tabular}{|c|} 
OFP \\
$\mathrm{mg} / \mathrm{km}$
\end{tabular} & $\begin{array}{l}\mathrm{NMHC} \\
\mathrm{mg} / \mathrm{km}\end{array}$ & \begin{tabular}{|c|} 
OFP \\
$\mathrm{mg} / \mathrm{km}$
\end{tabular} & \begin{tabular}{l|}
$\mathrm{NMHC}$ \\
$\mathrm{mg} / \mathrm{km}$ \\
\end{tabular} & $\begin{array}{c}\text { OFP } \\
\mathrm{mg} / \mathrm{km}\end{array}$ \\
\hline 2,2-Dimethyl-Hexan & VocAir & 1.02 & 0.000 & 0.000 & 0.000 & 0.000 & 0.000 & 0.000 & \begin{tabular}{|l|}
0.000 \\
\end{tabular} & 0.000 \\
\hline 2,4,4-Trimethyl-2-Penten & VocAir & 6.29 & 0.000 & 0.000 & 0.000 & 0.000 & 0.000 & 0.000 & 0.000 & 0.000 \\
\hline Ethyl-Cyclo-Pentan & VocAir & 2.01 & 0.000 & 0.000 & 0.000 & 0.000 & 0.000 & 0.000 & 0.000 & 0.000 \\
\hline 2,5-Dimethyl-Hexan & VocAir & 1.46 & 0.000 & 0.000 & 0.000 & 0.000 & 0.000 & 0.000 & 0.000 & 0.000 \\
\hline 2,4-Dimethyl-Hexan & VocAir & 1.73 & 0.000 & 0.000 & 0.000 & 0.000 & 0.000 & 0.000 & 0.000 & 0.000 \\
\hline 3,3-Dimethyl-Hexan & VocAir & 1.24 & 0.000 & 0.000 & 0.000 & 0.000 & 0.000 & 0.000 & 0.000 & 0.000 \\
\hline 2,3,4-Trimethyl-Pentan & VocAir & 1.03 & 0.122 & 0.126 & 0.162 & 0.166 & 0.011 & 0.011 & 0.014 & 0.014 \\
\hline 2,3,3-Trimethyl-Pentan & VocAir & 1.02 & 0.000 & 0.000 & 0.000 & 0.000 & 0.000 & 0.000 & 0.000 & 0.000 \\
\hline Toluol & VocAir & 4.00 & 0.452 & 1.807 & 0.153 & 0.612 & 0.008 & 0.032 & 0.024 & 0.096 \\
\hline 2,3-Dimethyl-Hexan & VocAir & 1.19 & 0.000 & 0.000 & 0.000 & 0.000 & 0.000 & 0.000 & 0.000 & 0.000 \\
\hline 2-Methyl-Heptan & VocAir & 1.07 & 0.000 & 0.000 & 0.000 & 0.000 & 0.000 & 0.000 & 0.000 & 0.000 \\
\hline 4-Methyl-Heptan & VocAir & 1.25 & 0.000 & 0.000 & 0.000 & 0.000 & 0.000 & 0.000 & 0.000 & 0.000 \\
\hline 3-Methyl-Heptan & VocAir & 1.24 & 0.092 & 0.114 & 0.264 & 0.327 & 0.031 & 0.039 & 0.038 & 0.048 \\
\hline 1,2,3-Trimethyl-Cyclo-Pentan & VocAir & 1.63 & 0.000 & 0.000 & 0.000 & 0.000 & 0.000 & 0.000 & 0.000 & 0.000 \\
\hline cis-1,3-Dimethyl-Cyclo-Hexan & VocAir & 1.52 & 0.000 & 0.000 & 0.000 & 0.000 & 0.000 & 0.000 & 0.000 & 0.000 \\
\hline trans-1,4-Dimethyl-Cyclo-Hexan & VocAir & 1.47 & 0.000 & 0.000 & 0.000 & 0.000 & 0.000 & 0.000 & 0.000 & 0.000 \\
\hline 2,2,5-Trimethyl-Hexan & VocAir & 1.13 & 0.195 & 0.220 & 0.346 & 0.391 & 0.000 & 0.000 & 0.066 & 0.075 \\
\hline 1-Octen & VocAir & 3.25 & 0.000 & 0.000 & 0.000 & 0.000 & 0.000 & 0.000 & 0.000 & 0.000 \\
\hline trans-4-Octen & VocAir & 4.81 & 0.000 & 0.000 & 0.000 & 0.000 & 0.000 & 0.000 & 0.000 & 0.000 \\
\hline n-Octan & VocAir & 0.90 & 0.111 & 0.100 & 0.382 & 0.344 & 0.021 & 0.019 & 0.000 & 0.000 \\
\hline trans-2-Octen & VocAir & 6.00 & 0.000 & 0.000 & 0.000 & 0.000 & 0.000 & 0.000 & 0.000 & 0.000 \\
\hline trans-1,3-Dimethyl-Cyclo-Hexan & VocAir & 1.52 & 0.000 & 0.000 & 0.000 & 0.000 & 0.000 & 0.000 & 0.000 & 0.000 \\
\hline cis-2-Octen & VocAir & 4.81 & 0.000 & 0.000 & 0.000 & 0.000 & 0.000 & 0.000 & 0.000 & 0.000 \\
\hline 2,3,5-Trimethyl-Hexan & VocAir & 1.22 & 0.000 & 0.000 & 0.000 & 0.000 & 0.000 & 0.000 & 0.000 & 0.000 \\
\hline 2,4-Dimethyl-Heptan & VocAir & 1.38 & 0.000 & 0.000 & 0.000 & 0.000 & 0.000 & 0.000 & 0.000 & 0.000 \\
\hline cis-1,2-Dimethyl-Cyclo-Hexan & VocAir & 1.41 & 0.000 & 0.000 & 0.000 & 0.000 & 0.000 & 0.000 & 0.000 & 0.000 \\
\hline Ethyl-Cyclo-Hexan & VocAir & 1.47 & 0.000 & 0.000 & 0.000 & 0.000 & 0.000 & 0.000 & 0.000 & 0.000 \\
\hline 3,5-Dimethyl-Heptan & VocAir & 1.56 & 0.000 & 0.000 & 0.000 & 0.000 & 0.000 & 0.000 & 0.000 & 0.000 \\
\hline Ethyl-Benzol & VocAir & 3.04 & 0.190 & 0.578 & 0.260 & 0.790 & 0.037 & 0.114 & 0.017 & 0.052 \\
\hline 2,3-Dimethyl-Heptan & VocAir & 1.09 & 0.000 & 0.000 & 0.000 & 0.000 & 0.000 & 0.000 & 0.000 & 0.000 \\
\hline m,p-Xylol & VocAir & 8.45 & 0.052 & 0.440 & 0.048 & 0.407 & 0.003 & 0.028 & 0.000 & 0.000 \\
\hline 4-Methyl-Octan & VocAir & 0.95 & 0.000 & 0.000 & 0.000 & 0.000 & 0.000 & 0.000 & 0.000 & 0.000 \\
\hline 2-Methyl-Octan & VocAir & 0.83 & 0.000 & 0.000 & 0.000 & 0.000 & 0.000 & 0.000 & 0.000 & 0.000 \\
\hline 3-Methyl-Octan & VocAir & 0.99 & 0.000 & 0.000 & 0.000 & 0.000 & 0.000 & 0.000 & 0.000 & 0.000 \\
\hline Ethenyl-Benzol & VocAir & 1.73 & 0.060 & 0.104 & 0.097 & 0.167 & 0.018 & 0.031 & 0.011 & 0.019 \\
\hline o-Xylol & VocAir & 7.64 & 0.051 & 0.390 & 0.067 & 0.514 & 0.006 & 0.049 & 0.003 & 0.023 \\
\hline
\end{tabular}




\section{PARTE 4}

\begin{tabular}{|c|c|c|c|c|c|c|c|c|c|c|}
\hline \multirow[b]{2}{*}{$\begin{array}{l}\text { Hidrocarbonetos } \\
\text { (C2-C12) }\end{array}$} & \multirow[b]{2}{*}{$\begin{array}{c}\text { Mess- } \\
\text { verfahren }\end{array}$} & \multirow[b]{2}{*}{ MIR } & \multicolumn{2}{|c|}{ 0404-E22 } & \multicolumn{2}{|c|}{ 0405-E22 } & \multicolumn{2}{|c|}{ 0410-E100 } & \multicolumn{2}{|c|}{ 0411-E100 } \\
\hline & & & $\begin{array}{l}\text { NMHC } \\
\mathrm{mg} / \mathrm{km}\end{array}$ & \begin{tabular}{|c|} 
OFP \\
$\mathrm{mg} / \mathrm{km}$
\end{tabular} & $\begin{array}{l}\mathrm{NMHC} \\
\mathrm{mg} / \mathrm{km}\end{array}$ & \begin{tabular}{|c|} 
OFP \\
$\mathrm{mg} / \mathbf{k m}$
\end{tabular} & $\begin{array}{l}\mathrm{NMHC} \\
\mathrm{mg} / \mathrm{km}\end{array}$ & \begin{tabular}{|c|} 
OFP \\
$\mathrm{mg} / \mathrm{km}$
\end{tabular} & \begin{tabular}{l|}
$\mathrm{NMHC}$ \\
$\mathrm{mg} / \mathrm{km}$ \\
\end{tabular} & $\begin{array}{c}\text { OFP } \\
\mathrm{mg} / \mathrm{km}\end{array}$ \\
\hline 1-Nonen & VocAir & 2.60 & 0.000 & 0.000 & 0.000 & 0.000 & 0.000 & 0.000 & 0.000 & 0.000 \\
\hline n-Nonan & VocAir & 0.78 & 0.076 & 0.059 & 0.104 & 0.081 & 0.004 & 0.003 & 0.001 & 0.001 \\
\hline (1-Methyl-ethyl)-Benzol & VocAir & 2.52 & 0.017 & 0.042 & 0.024 & 0.062 & 0.035 & 0.089 & 0.000 & 0.000 \\
\hline 2,2-Dimethyl-Octan & VocAir & 0.83 & 0.000 & 0.000 & 0.000 & 0.000 & 0.000 & 0.000 & 0.000 & 0.000 \\
\hline 2,4-Dimethyl-Octan & VocAir & 1.03 & 0.000 & 0.000 & 0.000 & 0.000 & 0.000 & 0.000 & 0.000 & 0.000 \\
\hline n-Propyl-Benzol & VocAir & 2.03 & 0.101 & 0.204 & 0.206 & 0.418 & 0.151 & 0.306 & 0.221 & 0.449 \\
\hline 1-Methyl-3-ethyl-Benzol & VocAir & 7.39 & 0.060 & 0.442 & 0.053 & 0.394 & 0.004 & 0.027 & 0.031 & 0.233 \\
\hline 1-Methyl-4-ethyl-Benzol & VocAir & 4.44 & 0.042 & 0.186 & 0.008 & 0.035 & 0.003 & 0.014 & 0.000 & 0.000 \\
\hline 1,3,5-Trimethyl-Benzol & VocAir & 11.76 & 0.081 & 0.955 & 0.164 & 1.932 & 0.055 & 0.648 & 0.000 & 0.000 \\
\hline 1-Methyl-2-ethyl-Benzol & VocAir & 5.59 & 0.311 & 1.739 & 0.337 & 1.886 & 0.062 & 0.349 & 0.028 & 0.159 \\
\hline n-Decan & VocAir & 0.68 & 0.058 & 0.040 & 0.087 & 0.059 & 0.072 & 0.049 & 0.041 & 0.028 \\
\hline (2-Methyl-propyl)-Benzol & VocAir & 2.36 & 056 & 0.133 & 0.122 & 0.289 & 0.057 & .134 & 0.063 & 0.150 \\
\hline (1-Methyl-propyl)-Benzol & MS-Therm & 2.36 & 0.000 & 0.000 & 0.000 & 0.000 & 0.000 & 0.000 & 0.000 & 0.000 \\
\hline 1-Methyl-3-(1-methyl-ethyl)-Benzol & MS-Therm & 7.10 & 0.000 & 0.000 & 0.000 & 0.000 & 0.000 & 0.000 & 0.000 & 0.000 \\
\hline 1,2,3-Trimethyl-Benzol & VocAir & 11.97 & 0.050 & 0.598 & 0.058 & 0.696 & 0.039 & 0.471 & 0.049 & 0.590 \\
\hline 1-Methyl-4-(1-methyl-ethyl) & MS-Therm & 4.44 & 0.000 & 0.000 & 0.000 & 0.000 & 0.000 & 0.000 & 0.000 & 0.000 \\
\hline Indan & MS-Therm & 3.32 & 0.000 & 0.000 & 0.000 & 0.000 & 0.000 & 0.000 & 0.000 & 0.000 \\
\hline 1-Methy & MS-Therm & 5.4 & 0.000 & 0.000 & 0.000 & 0.000 & 0.000 & 0.000 & 0.000 & 0.000 \\
\hline 1,3-Dieth & VocAir & 7.1 & 0.131 & 0.928 & 0.045 & 0.321 & 0.005 & 0.036 & 0.016 & 0.117 \\
\hline 1,4-Dieth & VocAir & 4.4 & 0.137 & 0.608 & 0.021 & 0.091 & 0.014 & 0.061 & 0.019 & 0.085 \\
\hline 1-Methyl-3-n-propyl-Benzol & MS-Therm & 7.1 & 0.000 & 0.000 & 0.000 & 0.000 & 0.000 & 0.000 & 0.000 & 0.000 \\
\hline 1-Methyl-4-n-p & MS-Therm & 4.4 & 0.000 & 0.000 & 0.000 & 0.000 & 0.000 & 0.000 & 0.000 & 0.000 \\
\hline 1,2-Diethyl-Benzol & VocAir & 5.49 & 0.024 & 0.133 & 0.035 & 0.195 & 0.019 & 0.103 & 0.026 & 0.143 \\
\hline 1-Methyl-2-n-propyl-BeI & MS-Therm & 5.49 & 0.000 & 0.000 & 0.000 & 0.000 & 0.000 & 0.000 & 0.000 & 0.000 \\
\hline 1,4-Dimethyl-2-ethyl-Benzol & MS-Therm & 7.55 & 0.000 & 0.000 & 0.000 & 0.000 & 0.000 & 0.000 & 0.000 & 0.000 \\
\hline 1,3-Dimethyl-4-ethyl-Benzol & MS-Therm & 7.55 & 0.000 & 0.000 & 0.000 & 0.000 & 0.000 & 0.000 & 0.000 & 0.000 \\
\hline 1,2-Dimethyl-4-ethyl-Benzol & MS-Therm & 7.55 & 0.000 & 0.000 & 0.000 & 0.000 & 0.000 & 0.000 & 0.000 & 0.000 \\
\hline 1,3-Dimethyl- & MS-Therm & 10.15 & 0.000 & 0.000 & 0.000 & 0. & 0.000 & 0.000 & 0.000 & 0.000 \\
\hline n-Undecan & VocAir & 0.61 & 0.027 & 0.017 & 0.031 & 0.019 & 0.002 & 0.001 & 0.002 & 0.001 \\
\hline $1,2-[$ & MS-Therm & 10.15 & 0.000 & 0.000 & 0.000 & 0.000 & 0.000 & 0.000 & 0.000 & 0.000 \\
\hline 1,2,4,5-Tetramethyl-Benzol & MS-Therm & 9.26 & 0.000 & 0.000 & 0.000 & 0.000 & 0.000 & 0.000 & 0.000 & 0.000 \\
\hline 1,2,3,5-Tetrameth & VocAir & 9.2 & 0.000 & 0.000 & 0.000 & 0.000 & 0.000 & 0.000 & 0.000 & 0.000 \\
\hline 1-Methyl-2-n & MS-Therm & 4.7 & 0.000 & 0.000 & 0.000 & 0.000 & 0.000 & 0.000 & 0.000 & 0.000 \\
\hline 1-(1,1-Dimethyl-ethyl)-2-methyl-Benzol & MS-Therm & 4.73 & 0.000 & 0.000 & 0.000 & 0.000 & 0.000 & 0.000 & 0.000 & 0.000 \\
\hline 1,2,3,4-Tetramethyl-Benzol + n-Pentyl-Benzo & VocAir & 9.26 & 0.005 & 0.051 & 0.015 & 0.139 & 0.016 & 0.144 & 0.006 & 0.055 \\
\hline n-Pentyl-Benzol & MS-Therm & 2.12 & 0.000 & 0.000 & 0.000 & 0.000 & 0.000 & 0.000 & 0.000 & 0.000 \\
\hline 1-(1,1-Dimethyl-ethyl)-3,5-dimethyl-Benzol & MS-Therm & 8.02 & 0.000 & 0.000 & 0.000 & 0.000 & 0.000 & 0.000 & 0.000 & 0.000 \\
\hline Naphthalin & MS-Therm & 3.34 & 0.000 & 0.000 & 0.000 & 0.000 & 0.000 & 0.000 & 0.000 & 0.000 \\
\hline n-Dodecan & VocAir & 0.55 & 0.000 & 0.000 & 0.031 & 0.017 & 0.000 & 0.000 & 0.000 & 0.000 \\
\hline 1,3,5-Triet & VocAir & 7.50 & 0.002 & 0.012 & 0.011 & 0.085 & 0.009 & 0.065 & 0.009 & 0.065 \\
\hline Total de Hidrocarbone & & & 16.214 & 79.558 & 19.262 & 88.711 & \begin{tabular}{|l|l|}
19.135 \\
\end{tabular} & 66.650 & \begin{tabular}{|l|l}
17.215 \\
\end{tabular} & 48.921 \\
\hline
\end{tabular}




\subsection{RESUMO DOS RESULTADOS DAS MEDIÇõES DE NMHC E CÁLCULO DA REATIVIDADE ESPECÍFICA COMPARATIVA (E100 x E22)}

\begin{tabular}{|c|c|c|c|c|}
\hline Combustível & \multicolumn{2}{|c|}{ E22 } & \multicolumn{2}{|c|}{ E100 } \\
\hline Teste $\mathbf{n} \stackrel{\circ}{ }$ & 0404-E22 & 0405-E22 & 0410-E100 & 0411-E100 \\
\hline Somatória de NMHC (mg/km)(151 tipos de HC) & 16.214 & 19.262 & 19.135 & 17.215 \\
\hline Média & \multicolumn{2}{|c|}{17.738} & \multicolumn{2}{|c|}{18.175} \\
\hline Somatória de OFP (mg/km x MIR) 151 tipos de HC & 79.558 & 88.711 & 66.65 & 48.921 \\
\hline Média & \multicolumn{2}{|c|}{84.135} & \multicolumn{2}{|c|}{57.786} \\
\hline Reatividade específica (OFP/NMHC) & \multicolumn{2}{|c|}{4.7} & \multicolumn{2}{|c|}{3.2} \\
\hline Alteração\% (E100 x E22) & \multicolumn{4}{|c|}{-32.0} \\
\hline
\end{tabular}

\section{CONCLUSÃO:}

A reatividade específica do potencial de formação de ozônio atmosférico das emissões de hidrocarbonetos não metano (NMHC), do Polo BlueMotion 1.6 L Eflex emitidas pelo gás de escapamento, quando o veículo é abastecido com 100\% de etanol hidratado(E100) é 32\% inferior a reatividade específica das emissões de NMHC no mesmo veículo, quando abastecido com gasolina C22 (E22). 


\section{ANEXO I - DEFINIÇÕES}

Para este trabalho foram adotadas algumas definições e parte do conceito do California NONMethane Organic Gas Test procedures - March 22, 2012 do CARB (California Air Resources Board) para possibilitar o cálculo da reatividade especifica dos hidrocarbonetos não metano:

$\mathrm{OFP}=\sum \mathrm{NMHC} * \mathrm{MIR}$

$\mathrm{SR}=\Sigma \mathrm{OFP} / \Sigma \mathrm{NMHC}$

Sendo que,

$\mathrm{MIR}=$ Maximum Incremental Reactivity

OFP $=$ Potencial Forming Ozone

$\mathrm{SR}=$ Specific Reactivity 


\section{TABELA № 1 - CARACTERÍSTICAS FÍSICO QUÍMICAS DA GASOLINA C22 UTILIZADA NOS ENSAIOS NA ALEMANHA}

\begin{tabular}{|c|c|c|c|c|}
\hline Propriedades & Unidade & Método de ensaio & $\begin{array}{c}\text { Especificação ANP } \\
57 / 2011\end{array}$ & $\begin{array}{c}\text { Resultados extraídos do } \\
\text { certificado RPBC 3386- } \\
12 \mathrm{~S} \\
\end{array}$ \\
\hline Periodo de indução & minutos & ASTM D 525 & $\min .360$ & $>720$ \\
\hline Goma atual & $\mathrm{mg} / 100 \mathrm{~mL}$ & ASTM D 381 & $\max .5$ & $<1$ \\
\hline MON & - & ASTM D 2700 & $\min .82$ & 83.3 \\
\hline RON & - & ASTM D 2699 & - & 96.3 \\
\hline MON + RON /2 & - & ASTM D 2699 & $\min .87$ & 89.3 \\
\hline 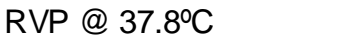 & $\mathrm{kPa}$ & ASTM D 5191 & 45 a 69 & 62.9 \\
\hline Densidade@20ㄷ & $\mathrm{Kg} / \mathrm{m}^{3}$ & ASTM D 4052 & anotar & 751.6 \\
\hline Destilação & - & ASTM D 86 & - & - \\
\hline Ponto inicial de ebulição & ${ }^{\circ} \mathrm{C}$ & ASTM D 86 & - & 40.2 \\
\hline $10 \%$ evaporado & $\stackrel{\circ}{ } \mathrm{C}$ & ASTM D 86 & $\max .65$ & 55.5 \\
\hline $50 \%$ evaporado & ${ }^{\circ} \mathrm{C}$ & ASTM D 86 & $\max .80$ & 72.3 \\
\hline $90 \%$ evaporado & $\stackrel{\circ}{ } \mathrm{C}$ & ASTM D 86 & $\max .190$ & 175.3 \\
\hline Ponto final de ebulição & ${ }^{\circ} \mathrm{C}$ & ASTM D 86 & $\max .220$ & 212.5 \\
\hline Resíduo & vol \% & ASTM D 86 & $\max .2$ & 0.6 \\
\hline Etanol & vol \% & NBR 13992 & $\max .25$ & 22 \\
\hline $\mathrm{HC}$ aromáticos & vol \% & ASTM D 1319 & $\max .45$ & 20.4 \\
\hline HC olefínicos & vol \% & ASTMD 1319 & $\max .30$ & 21.1 \\
\hline Benzeno & vol \% & ASTM D 36016 & $\max .1 .0$ & 0.22 \\
\hline Enxôfre & $\mathrm{mg} / \mathrm{kg}$ & ASTM D 4295 & $\max .800$ & 289 \\
\hline
\end{tabular}




\section{TABELA № 2 - CARACTERÍSTICAS FÍSICO QUÍMICAS DO ETANOL HIDRATADO UTILIZADO NOS ENSAIOS NA ALEMANHA}

\begin{tabular}{|c|c|c|c|c|}
\hline Propriedades & Unidade & $\begin{array}{l}\text { Método de } \\
\text { ensaio }\end{array}$ & $\begin{array}{c}\text { Especificão ANP } \\
7 / 2011\end{array}$ & $\begin{array}{l}\text { Resultados extraídos do } \\
\text { certificado lote } 257\end{array}$ \\
\hline Coloração & - & Visual & colorness & incolor \\
\hline Aparência & - & Visual & $\begin{array}{l}\text { Limpo e isento de } \\
\text { impurezas }\end{array}$ & Limpo e isento de impurezas \\
\hline Densidade @ 20ㄷ & $\mathrm{kg} / \mathrm{m}^{3}$ & NBR 5592 & 807.6 a 811.0 & 808.1 \\
\hline $\begin{array}{l}\text { Acidez total(expresso } \\
\text { como ácido acético) }\end{array}$ & $\mathrm{mg} / \mathrm{L}$ & NBR 9866 & $\max .30$ & 7.2 \\
\hline $\mathrm{pH}$ & - & NBR 10891 & 6 to 8 & 6.6 \\
\hline Teor de sulfatos & $\mathrm{mg} / \mathrm{kg}$ & NBR 10894 & $\max .4$ & $<1$ \\
\hline Teor de sódio & $\mathrm{mg} / \mathrm{kg}$ & NBR 10422 & $\max .2$ & 0.18 \\
\hline Condutividade Elétrica & $\mu S / m$ & NBR 10547 & 350 & 8 \\
\hline Teor de Etanol & $\%$ Vol & NBR15639 & 95.1 to 96.0 & 95.8 \\
\hline Teor de Etanol & $\% \mathrm{~m}$ & NBR 15639 & 92.5 to 93.8 & 93.6 \\
\hline $\begin{array}{l}\text { Resíduo por } \\
\text { evaporação }\end{array}$ & $\mathrm{mg} / 100 \mathrm{~mL}$ & NBR 8644 & 5 & 0.5 \\
\hline
\end{tabular}




\section{REFERÊNCIAS BIBLIOGRÁFICAS:}

1) California NON-Methane Organic Gas Test procedures - March 22, 2012) do CARB (California Air Resources Board);

2) Paper do Environment Canada "Mobile Source Emissions\& Biofuels: An Overview of Selected Canadian Federal R\&D". 